\title{
CLOSEST SOUTHEAST SUBMATRIX THAT MAKES MULTIPLE A DEFECTIVE EIGENVALUE OF THE NORTHWEST ONE
}

\author{
Gorka Armentia, Juan-Miguel Gracia and Francisco E. Velasco
}

Abstract. Given three complex matrices $A \in \mathbb{C}^{n \times n}, B \in \mathbb{C}^{n \times m}$ and $C \in \mathbb{C}^{m \times n}$, and given a defective eigenvalue $z_{0}$ of $A$, we study when the set $\mathcal{S}$ of matrices $X \in \mathbb{C}^{m \times m}$ such that $z_{0}$ is a multiple eigenvalue of the matrix

$$
\left(\begin{array}{ll}
A & B \\
C & X
\end{array}\right) .
$$

is nonempty. Moreover, when $\mathcal{S} \neq \emptyset$, given a fourth matrix $D \in \mathbb{C}^{m \times m}$ we find a matrix $X_{0} \in \mathcal{S}$ such that

$$
\left\|X_{0}-D\right\|=\min \{\|X-D\|: X \in \mathcal{S}\} .
$$

Mathematics subject classification (2010): 15A18, 15A60, 15A09, 93B10.

Keywords and phrases: Nearest matrix, multiple eigenvalues, controllable, observable, Moore-Penrose, surjective mapping theorem, nonsemisimple.

\section{REFERENCES}

[1] R. G. Bartle, The Elements of Real Analysis, Second Edition, Wiley, New York, 1976.

[2] S. L. Campbell and C. D. Meyer, Generalized Inverses of Linear Transformations, Pitman, London, 1979.

[3] J. DEMmeL, The smallest perturbation of a submatrix which lowers the rank and constrained total least squares problems, SIAM J. Numer. Anal., 24 (1) (1987) 199-206,

[4] J. M. GonzÁLEZ de DURAnA AND J. M. GRACIA, Geometric multiplicity margin for a submatrix, Linear Algebra Appl., 349 (2002) 77-104.

[5] J. M. GRACIA AND F. E. VELASCO, Nearest southeast submatrix that makes multiple a prescribed eigenvalue, Part 1, Linear Algebra Appl., 430 (2009) 1196-1215.

[6] J. M. GRACIA AND F. E. VELASCO, Nearest southeast submatrix that makes multiple an eigenvalue of the normal northwest submatrix, Operators and Matrices, 430 (1) (2012) 1-35.

[7] J. R. Magnus And H. Neudecker, Matrix Differential Calculus with Applications in Statistics and Econometrics, Revised Edition, John Wiley and Sons, New York, 1999.

[8] G. Mars aglia And G. P. Styan, Equalities and inequalities for ranks of matrices, Linear Multilinear Algebra, 2 (1974) 269-292.

[9] G. S. Rogers, Matrix Derivatives, Lectures Notes in Statistics, Vol. 2, Marcel Dekker, Inc., New York, 1980.

[10] W. WASow, Linear Turning Point Theory, Springer-Verlag, Berlin, 1985.

[11] M. WEI, Perturbation theory for the Eckart-Young-Mirsky theorem and the constrained total least squares problem, Linear Algebra Appl., 280 (1998) 267-287. 